\title{
Undergraduate audiology students' perceived competence and confidence in conducting otoscopic examination following video otoscopic training
}

Ben Sebothoma* (10) and Katijah Khoza-Shangased

\begin{abstract}
Background: Emerging research indicates that video otoscopy can be used as a teaching tool to enhance students' ability to identify outer and middle ear pathologies. However, there is little research on the perceptions of audiology students regarding their competence and confidence following video otoscopic training, and how they view the use of video otoscopy as a teaching tool. Therefore, this study aimed to determine undergraduate (UG) audiology students' perceived competence and confidence in conducting otoscopy following training by video otoscopic examination.
\end{abstract}

Methods: A survey methodology with a cross sectional design was employed. An electronic questionnaire was distributed to all third and fourth year (senior) $(N=79)$ UG audiology students using Survey Monkey. Ethical approval and permission from relevant stakeholders were obtained. Data were analysed using both descriptive and inferential statistics.

Results: $60 \%$ of the students felt competent in performing otoscopy, while $63.3 \%$ felt less competent in interpreting otoscopic examination findings. $43.3 \%$ felt they can confidently and competently identify outer ear pathologies. There was no association between the number of video otoscopic examinations performed and perceived competence or/and confidence. There was also no statistically significant relationship between year of study (e.g., third year versus fourth year) and perceived competence or/and confident ( $p=0.7131)$. Almost all (97\%) students felt that video otoscopic training should continue to be part of the clinical training as it helped them enhance their skills in performing otoscopy.

Conclusions: Current findings highlight the need to improve students' practical training, incorporating pathologic ears into the curriculum. These findings also highlight the importance of supplementing practical training methodologies with changing technological advancements, particularly where tele-audiology opportunities may exist.

Keywords: Confidence, Competence, Students, Training, Video otoscopy

\footnotetext{
* Correspondence: Ben.Sebothoma@wits.ac.za

Department of Speech pathology and Audiology, University of the

Witwatersrand, Private Bag 3, Wits, 2050, Johannesburg, South Africa
}

(C) The Author(s). 2021 Open Access This article is licensed under a Creative Commons Attribution 4.0 International License, which permits use, sharing, adaptation, distribution and reproduction in any medium or format, as long as you give appropriate credit to the original author(s) and the source, provide a link to the Creative Commons licence, and indicate if changes were made. The images or other third party material in this article are included in the article's Creative Commons licence, unless indicated otherwise in a credit line to the material. If material is not included in the article's Creative Commons licence and your intended use is not permitted by statutory regulation or exceeds the permitted use, you will need to obtain permission directly from the copyright holder. To view a copy of this licence, visit http://creativecommons.org/licenses/by/4.0/ The Creative Commons Public Domain Dedication waiver (http://creativecommons.org/publicdomain/zero/1.0/) applies to the data made available in this article, unless otherwise stated in a credit line to the data. 


\section{Background}

Otoscopic examination forms an important part of the standard audiological test battery for diagnosis of auditory disorders [1]; specifically, outer and middle ear disorders [2]. Yet, many students in hearing health programmes are not competent and confident in conducting otoscopic examinations $[3,4,2]$. The lack of competence and confidence in conducting otoscopic examinations may have clinical implications, leading to misdiagnosis of the outer and middle ear pathologies [5], which may be costly [6].

Literature has indicated that misdiagnosis of outer or/ and middle ear pathologies may lead to a myriad of complications. These complications include, but are not limited to, permanent hearing loss, cognitive difficulties, and intracranial complications [7-9]. If these conditions are not treated timeously, they can affect the individuals' communication and overall quality of life [10, 11]. To improve students' abilities to identify early signs of outer and middle ear pathologies and reduce the likelihood of misdiagnosis, it is crucial to equip them with the necessary otoscopic examination skills. This is even more crucial in low-and-middle-income countries (LMICs) where hearing health resources are extremely limited and preventive ear and hearing care is crucial [12].

Several studies have been conducted to investigate the competence and confidence of students' abilities to conduct, analyse and interpret otoscopic examinations $[3,13,2]$. These studies have used simulators to train and assess students' abilities to analyse normal versus abnormal structures of the outer and middle ear; and significant improvement in performance was found following training. Although simulators may be useful in training students' skills, simulators may not be available in resource limited countries, such as South Africa, due to their costs [14]. An alternative additional training method [4] is therefore required to ensure that students demonstrate competence in conducting, analysing, and interpreting otoscopic results before they graduate [15].

A video otoscope is a recent technological advancement and easy to use tool to examine the outer and middle ear structures $[16,17]$. It typically uses a speculum that channels light, incorporates a video camera into the system, and allows for the transmission of images onto a screen [1], with visuals of typically the external auditory meatus (EAM) and the tympanic membrane (TM) [18]. Due to the added advantages illustrated above, Jaisinghani and colleagues [19] argue that video otoscopy can be used to effectively teach the anatomy and pathology of the EAM and the TM. Structural changes in the EAM and of the TM can provide valuable information about middle ear pathologies such as otitis media [20]. However, there is little research in South Africa in this area.
Audiology students in the department of Speech Pathology and Audiology at the University of the Witwatersrand were trained to conduct, analyse and interpret otoscopic findings using video otoscopy. Students were also instructed to capture additional video otoscopy, analyse and interpret results as part of their class assignment. However, it was not known if the video otoscopic training and class assignment had improved their competence and confidence in conducting, analysing, and interpreting otoscopic results. Therefore, the present study aimed to describe the audiology students' perceived competence and confidence in conducting otoscopic examination following video otoscopic training.

\section{Research aim}

The main aim of this study was to explore audiology students' perceived competence and confidence in identifying outer and middle ear disorders following video otoscopic training, and to capture their views on video otoscopy as a training tool for ear visual inspection.

The specific objectives of the study were:

- To determine audiology students' perceived competence in identifying outer and middle ear pathologies following video otoscopic training.

- To determine audiology students' perceived confidence in identifying outer and middle ear pathologies following video otoscopic training.

- To describe audiology students' views on video otoscopy as a training tool.

\section{Method}

\section{Research design}

This study employed a cross-sectional survey design [21]. This research design was chosen because it allowed the researchers to obtain information from a cohort of students at a given point in time. All procedures in this study adhered to the World Medical Association (WMA) Declaration of Helsinki (2013) ethical guidelines. Ethical approval was obtained from the University of the Witwatersrand's Human Research Ethics committee (HREC) (non-medical) (Protocol Number: H19/09/ 49). Permission to conduct the study and to distribute the survey questionnaire to audiology students was obtained from the Head of the Audiology Department, and the University's Registrar at the University of the Witwatersrand, Johannesburg, South Africa.

\section{Data collection method}

Data collection was conducted using a self-administered online questionnaire. The questionnaire consisted of 17 Likert scale, multiple choice and closed and open ended questions, covering demographic information, perceived competence and confidence in performing otoscopic 
examination after video otoscopic training, as well as students' recommendations regarding this form of training. The questionnaire consisted mainly of close-ended questions, with an option of 'other' which allowed audiology students to provide additional information. The questionnaire was sent to 3 audiologists to comment on the content and clarity of the questionnaire, and to provide appropriate suggestions for improvements [21]. The questionnaire was emailed together with a suggestion form. The questionnaire was then piloted on 4 audiology students who met the inclusion criteria, who did not form part of the main study. The final version of the questionnaire was uploaded onto the Survey Monkey and distributed to all third $(\mathrm{n}=50)$ and fourth $(\mathrm{n}=29)$ year students by the professional administrative (PA) staff members of the department of Speech Pathology and Audiology. Data collection took 6 months to complete (March 2020 to August 2020). The survey was distributed to students at least 4 times to increase the response rate.

\section{Participants}

A non-probability purposive sampling was used to recruit and select participants [21]. Inclusion criteria included audiology students who underwent video otoscopic training. Participants in this study were 30 out of 79 UG audiology students (38\% response rate) at the University of the Witwatersrand, Johannesburg, South Africa. Prior to participating in this study, UG students had completed a 6-week revised course on anatomy, physiology, and pathology of the auditory system. This revision was then followed by a 3-week video otoscopic examination training. The training involved positioning of a patient, insertion of the speculum and channelling the light toward the tympanic membrane (TM) to obtain a clearer image, manoeuvring of the pinna when the TM cannot be visualized clearly, capturing of the image, saving of the image in a specific folder for this training, and analysing and interpreting the video otoscopic results. Students were also trained to identify any foreign objects such as occluded cerumen that may impede visualization of the tympanic membrane [18].

When training was completed, students were given a practical assignment to independently complete video otoscopic examination of 20 volunteer patients (40 ears each) and analyse the results. These volunteer patients were mainly university students from various training programmes or friends of students. Assignments were marked according to the following criteria: clarity of the captured images of the tympanic membrane, accuracy in interpreting all the tympanic membrane landmarks, and identification of pathologies if any existed. Constructive feedback was provided to students by the training facilitator.

\section{Data analysis}

Descriptive statistics [22] using Statistical Package for Social Science (SPSS) version 25 were used to analyse the data. Frequency tables and graphs were used to summarize the data. Inferential statistics such as chisquared were used to determine if there was any association between variables of interests such as year of study and competence/confidence, with p-value set at 0.05 . A proportional test was also used to determine if there was any association between students' performance during their assignment and perceived competence or/and confidence in conducting otoscopy.

\section{Results}

Thirty audiology students participated in this study. All students who participated in the study were female audiology students who were in third and fourth year of study (where fourth year is the final year of study). As part of the practical assignment requirement for video otoscopy, majority of the audiology students (93\%) completed about 10 to 20 (40 ears) video otoscopic assessments, while only $3 \%$ completed over 50 video otoscopic assessments (+100 ears). Figure 1 below illustrates the number of video otoscopic assessments completed by students.

Almost half (47\%) of the students indicated that they are somewhat confident, while $43 \%$ felt very competent in the overall otoscopic examination. Half $(50 \%)$ of the students felt confident, while $60 \%$ felt competent in performing otoscopic examination. $53 \%$ of the students felt less confident while $63 \%$ felt less competent in interpreting otoscopic examination. Further analysis showed that there was no statistical relationship between the number of video otoscopic examinations completed and perceived competence, $\mathrm{X}^{2}(6, N=30)=2.6374, p=0.8528$ or/and perceived confidence, $\mathrm{X}^{2}(8, N=30)=4.5153, p=$ 0.8079 . There was no statistically significant relationship between year of study (e.g., third year) and perceived confident, $\mathrm{X}^{2}(4, N=30)=2.12, p=0.7131$. There was also no statistically significant relationship between year of study and perceived competence in conducting otoscopic examination, $\mathrm{X}^{2}(3, N=30)=43126, p=0.2296$.

In order to determine whether there is any relationship between competence and students' performance during their assignment, four levels of competence were created (see Figs. 2 and 3). A proportion test based on these categories and student marks was conducted. The somewhat competent and very competent proportion test indicates that these are not significantly different, while the not so competent and the extremely competent groups indicated some significant difference. This means that the proportion of those who indicated to be somewhat competent were not significantly different from the proportion of those who scored 50-59\%, 


\section{Number of video otoscopy completed by} students $(\mathrm{N}=30)$

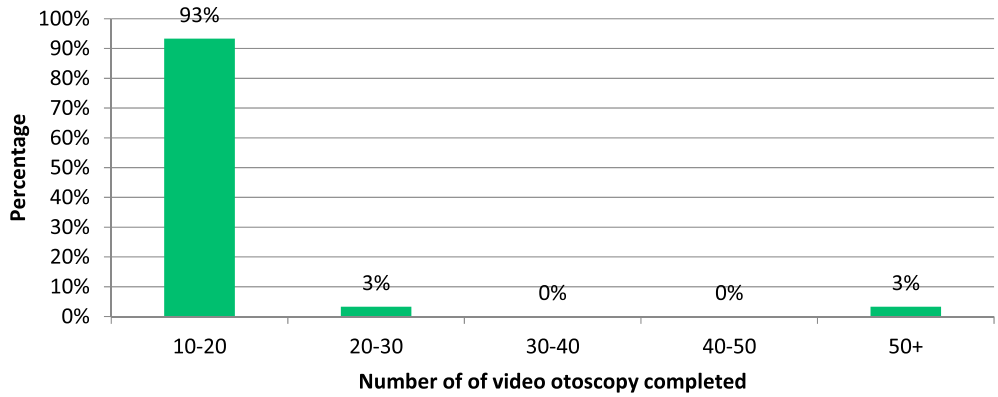

Fig. 1 Video otoscopy completed by audiology students

similarly the proportion of those who indicated to be very competent were not significantly different from those who scored $60-74 \%$. However, since these data are independent of each other, correlation and the strength of correlation could not be established.

In terms of pathologies that students could identify using otoscopy, just under half of the students (43\%) felt that they can identify outer ear pathologies, while $37 \%$ felt that they can identify both outer and middle ear pathologies.

Half of the students (50\%) felt that video otoscopic training helped them to become confident and competent in otoscopic examination, with the other half feeling that it had not helped them, and others feeling unsure. Participants who indicated that video otoscopic training helped them reported that this was due to the clear visualization of the tympanic membrane.

"You got a very clear and defined picture when using video otoscopy and it helped one become more comfortable at identifying the shape and bends of the ear canal, as well as the tympanic membrane" (participant 1).

"Tympanic membrane was more easily seen and interpretation for otoscopic results was much easier as well" (participant 2).

Participants who indicated that the video otoscopic training did not help them attributed this mainly to lack of time using the tool and machine dysfunction.

"Not having enough time with it because we had to share the device amongst students which takes time away from learning and mastering conducting and interpreting it" (Participant 3).

"The video otoscope did not function most of the time" (Participant 4).

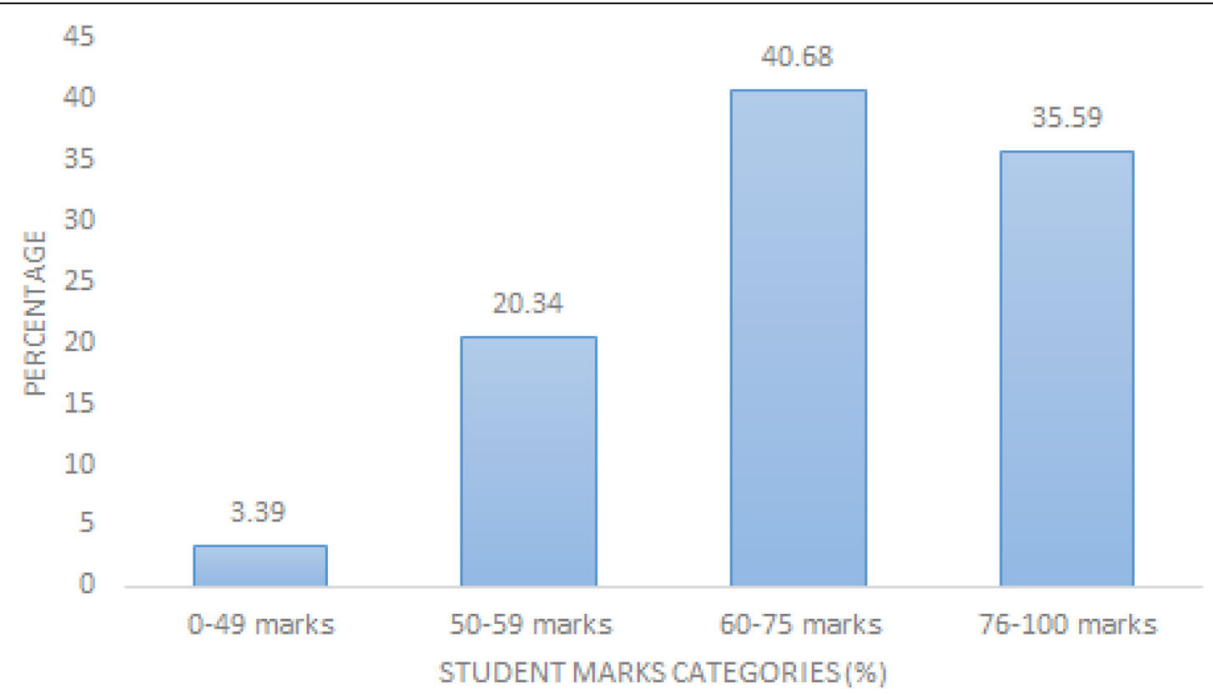

Fig. 2 Student assignment marks categories 


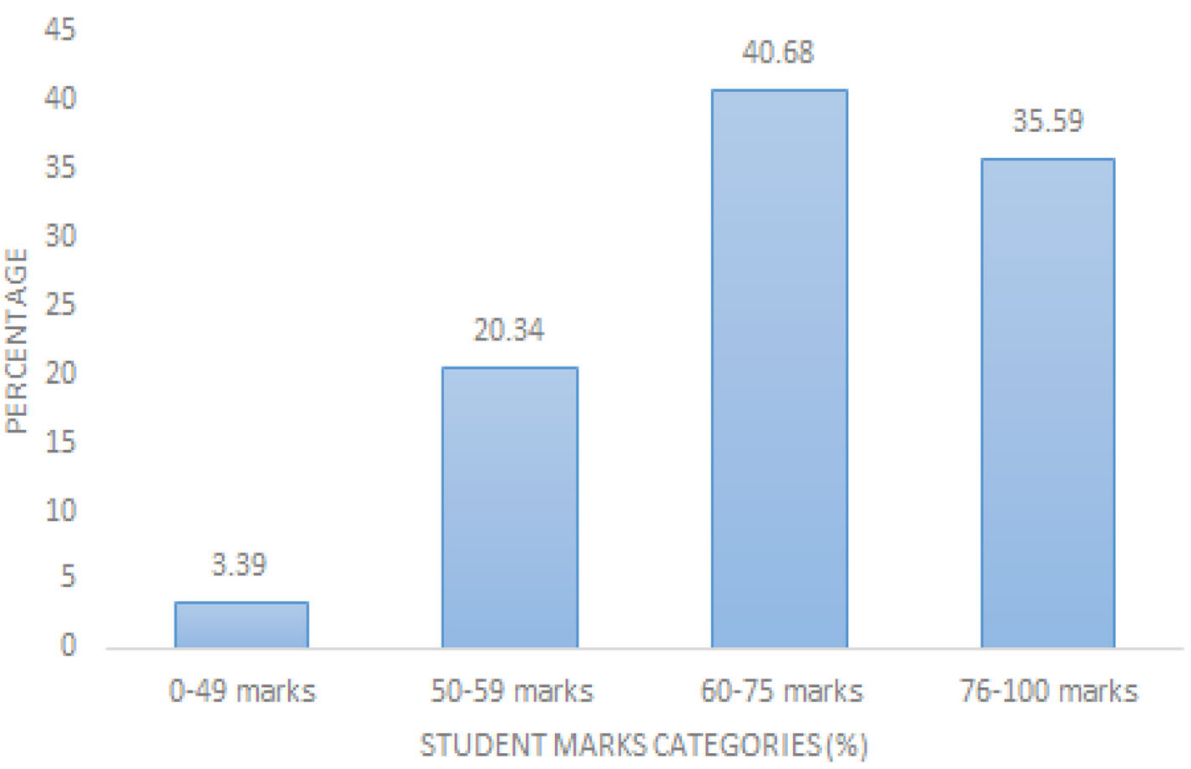

Fig. 3 Students' perception of competence in performing otoscopy

Despite this, almost all students (97\%) indicated that video otoscopic training should continue to be part of the students' clinical training.

\section{Discussion}

Otoscopic examination is an important audiological procedure that helps in identifying early signs of both outer and middle ear pathologies [13]. In fact, research has indicated that otoscopic examination can identify middle ear pathologies much better than the conventional tympanometry with single probe tone [18] that is used by audiologists. It is therefore crucial that students are equipped with the necessary skills to be competent and confident in conducting otoscopic examination. This study therefore sought to determine if audiology students perceived themselves as competent and/or confident in conducting otoscopic examination following video otoscopic training.

Current findings suggest that approximately $50 \%$ of the audiology students surveyed perceived themselves as competent and confident in conducting otoscopic examination, while the rest perceive themselves as less competent or/and confident. In terms of aspects of otoscopic examination that audiology students perceive themselves as less or more competent and confident in, majority of the students felt that they were less competent and confident in performing and interpreting otoscopic examination. The perceptions of audiology students in this study are inconsistent with previous studies that trained students [3, 13, 23]. Kaf and colleagues [13] found that majority of the audiology students (over $70 \%)$ perceived themselves to be more confident after training; while You and colleagues [23] also found that the perception of medical students regarding confidence in otoscopic examination significantly improved following training.

The difference between the current findings and previous studies can be attributed to different training methods used. Kaf and colleagues [13] used Mannikin ears that reflected various statuses of the middle ear, while in the current study audiology students used University students mainly with normal middle ear function. The lack of exposure to various pathologies during training could have played a role in students' perceptions. Students may be unsure on what they should be looking for when conducting otoscopic examination. Therefore, the findings of this study suggest a need for curriculum review for practical training of audiology students. Future training on otoscopic examination should consider incorporating patients with various middle ear pathologies.

In addition to video otoscopic training using volunteer patients, institution of higher learning, particularly in LMICs, should consider using simulations as part of clinical training as research suggests that simulations improve students' confidence and competence [24, 25]. Wilson and colleagues [25] argue that simulations should support clinical training and not replace clinical training. Von Buchwald and colleagues [26] found that junior doctors scored low in identifying simulated middle ear pathologies. These authors argue that the difficulty in identifying pathologies may have been due to lack of case history accompanying the pathologic ears. Therefore, in LMICs, training programmes for audiology students should ensure inclusion of case history information as part of training to help students with the 
identification of outer and middle ear pathologies, and to make sure that the training is not in isolation.

Regarding specific pathologies that students can confidently and/or competently identify using otoscopy, $43 \%$ of students felt that they can identify outer ear pathologies such as cerumen and foreign objects, while few indicated that they were able to identify outer and middle ear pathologies. These findings are also inconsistent with findings of a study conducted by Davies and colleagues [3] who found that $71 \%$ of medical students perceived their confidence in diagnosing pathologies of the ear to have improved following training. Although audiology students are not expected to diagnose pathologies of the ear, it is within the scope of audiology for students to distinguish normal from abnormal outer and middle ear pathologies in order to be able to plan further testing as well as appropriate intervention and/or referral [13]. Therefore, it is crucial that students' confidence and competence be improved for clinical bestpractice purposes. Davies and colleagues [3] argue that lack of confidence in diagnosing ear pathologies ultimately affects graduates in clinical practice.

Despite the students' lack of confidence and competence in identifying outer and middle ear, it was assuring that $97 \%$ of the students felt that video otoscopy should continue to be part of their clinical training. This means students feel that video otoscopic training may improve their confidence and competence if done properly. In studies by Kaf and colleagues [13] and Davies and colleagues [3], training included images of pathologic ears to help students distinguish between normal and abnormal. Therefore, further research in this area should ensure that pathologic ears are included in the training.

There is an increasing effort to develop mobile applications to facilitate identification and management of auditory and otolaryngological pathologies, including outer and middle ear pathologies [27]. Given the documented demand versus capacity challenge in LMICs, mobile applications can be used to help with the training of students to identify outer and middle ear pathologies, where instructors other than those physically available in local training institutions can be utilised. Bhavana and colleagues [28] found a higher sensitivity and specificity of smartphone otoscopy in identifying outer and middle ear pathologies when compared with oto-endoscopy. Myburgh and colleagues [29] developed an automated cloud-based smartphone system which uses image analysis to diagnose outer and middle ear pathologies. These mobile applications are crucial in LMICs and can be used to teach audiology students to be able to identify early signs of outer and middle ear pathologies; and can also facilitate peer and/or supervisor support though asynchronous tele-audiology modalities.
Given that the COVID-19 pandemic has severely affected the traditional face-to-face audiological service provision [30], including student training in institutions of higher learning, Khoza-Shangase and colleagues [31] emphasise the need for innovative models of service delivery and student training to ensure the continuity of care and student training during and beyond this pandemic. Telepractice and teletraining have been at the forefront to ensure continuity of care and student training. Bhavana and colleagues [28] argue that smartphone otoscopy can be used as a teaching tool within the telemedicine approach, especially in LMICs. Sebothoma and Khoza-Shangase [32] and Binol and colleagues [33] indicate that video otoscopic images of the tympanic membrane can be used within the telemedicine approach. Given the high sensitivity and specificity of video/digital otoscopy, this has important training and practice implications, particularly during the COVID-19 pandemic.

While the current study provides important evidence about the use of video otoscopy as a teaching tool for outer and middle ear pathologies, it is worth noting that at the time of this study, there was $100 \%$ female audiology students from third to fourth year at the University where the study was conducted. Although this gender profile may be slightly different in other universities, the predominant number of females in the cohort of students is not surprising given that the gender profile of the South African audiologists and speech therapists registered with the Health Professional Council of South Africa (HPCSA) is primarily (95\%) female [34]. These professions are highly feminised in South Africa, with efforts to transform the gender, linguistic and racial demographic profile afoot in the country. Despite this, it is not believed that this profile had an impact in the current findings. In addition, the sample size for this study was very small, with a response rate of $38 \%$ which is significantly lower than the ideal $60 \%$; and this certainly is believed to have had an impact on the current findings; and influences their generalizability. The impact of COVID-19 pandemic on data collection for this study highlighted the importance of enhancing teletraining and tele-data collection strategies in any training programme.

\section{Conclusions}

Audiology students in this study were somewhat confident and competent in conducting otoscopic examination following video otoscopic training. Further research is needed to determine if perception of students correlates to actual hands-on improvement of their otoscopic skills. This study also highlights the need for improving practical curriculum training of students in this area. Incorporating pathologic ears may be helpful in improving audiology students' confidence and competent 
in identifying outer and middle ear pathologies. This may help students to make appropriate referrals for medical management. Furthermore, this study also highlighted the need to supplement practical training methodologies with changing technological advancements; and enhancing tele-training and tele-data collection strategies going forward.

\section{Abbreviations}

COVID-19: Coronavirus disease 19; EAM: External auditory meatus; HREC: Human Research Ethics Committee; LMICs: Low- and middle-income countries; SPSS: Statistical Package for Social Science; PA: Professional administrators; TM: Tympanic membrane; UG: Undergraduate

\section{Acknowledgements}

Authors would like to thank audiology students who participated in this study. The authors would also like to thank the HOD of audiology, and the University registrar of the University of the Witwatersrand, Johannesburg for providing permission to conduct this study.

\section{Authors' contributions}

BS contributed to the conceptualisation of the study, design, developing the survey, collecting, analysis, writing and revising the manuscript. KKS contributed to the conceptualisation of the study, design, developing the survey, collecting, analysis, writing and revising the manuscript. All authors have read and approved the final manuscript.

\section{Funding}

The authors thank the National Institute for the Humanities and Social Sciences (NIHSS) for providing financial assistance for the publication of this manuscript.

\section{Availability of data and materials}

All data generated or analysed during this study are included in this published article.

\section{Declarations}

\section{Ethics approval and consent to participate}

All procedures in this study adhered to the World Medical Association (WMA) Declaration of Helsinki (2013) ethical guidelines. Ethical approval was obtained from the University of the Witwatersrand's Human Research Ethics committee (HREC) (non-medical) (Protocol Number: H19/09/49). Permission to conduct the study and to distribute the survey questionnaire to audiology students was obtained from the Head of the Audiology Department, and the University's Registrar at the University of the Witwatersrand, Johannesburg, South Africa. Participants gave written informed consent by clicking the button on the survey monkey after reading and understanding the participants' information sheet.

\section{Consent for publication}

Not applicable.

\section{Competing interests}

The authors declare that they have no financial or personal relationships that may have inappropriately influenced them in writing this article.

Received: 24 October 2020 Accepted: 1 September 2021

Published online: 27 September 2021

\section{References}

1. Kramer S, Brown DK. Audiology: Science to Practice. 3rd ed. San Diego, CA: Plural publishing. 2019

2. Lee NJ, Chae SM, Kim H, Lee JH, Min HJ, Park DE. Mobile-Based Video Learning Outcomes in Clinical Nursing Skill Education. A Randomized Controlled Trial. Comput Inform Nurs. 2016; 34: 8-15

3. Davies J, Djelic L, Campisi P, Forte V, Chiodo A. Otoscopy Simulation Training in a Classroom Setting: A Novel Approach to Teaching Otoscopy to Medical Students. Laryngoscope. 2014; 125: 2594-2597
4. Fisher WE, Pfeiderer AG. Is undergraduate otoscopy teaching adequate? An audit of clinical teaching. Journal of the Royal Society of Medicine. 1992; 85: $23-25$

5. British Society of Audiology. Recommended Procedure: Tympanometry. 2013. http://www.thebsa.org.uk/wp-content/uploads/2014/04/BSA_RP_ Tymp_Final_21Aug13_Final.pdf. Accessed 14 Jun 2020

6. Toman J, Moll A, Barnes M, Shenoi S, Porterfield JZ. The Role of Routine Culture in the Treatment of Chronic Suppurative Otitis Media: Implications for the Standard of Care in Rural Areas of South Africa. Trop. Med. Infect. Dis. 2019, 4, 10; doi:https://doi.org/10.3390/tropicalmed4010010

7. Kolo ES, Salisu AD, Yaro AM, Nwaorgu OGB. Sensorineural Hearing Loss in Patients with Chronic Suppurative Otitis Media. Indian J Otolaryngol Head Neck Surg. 2012; 64: 59-62

8. Olusanya BO, Okolo AA, Adeosun AA. Predictors of hearing loss in School entrants in developing country. J. Postgrad. Med. 2004; 50: 173-179

9. Sugiura S, Yasue M, Sakurai T, Sumigaki C, Uchida Y, Nakashia T, Toba K. Effect of cerumen impaction on hearing and cognitive functions in Japanese Older adults with cognitive impairment. Geriatr Gerontol Int. 2014; Suppl. 2: 56-61

10. Anderson S, Paebery-Clark A, White-Schwoch T, Drehobl S, Kraus N. Effects of hearing loss on the subcortical representation of speech cues. J Acoust Soc Am. 2013; 133: 3030-8

11. Dalton DS, Cruickshanks KJ, Klein BEK, Klein R, Wiley T, Nondahl DM. The Impact of Hearing Loss on Quality of Life in Older Adults. Gerontologist. 2003;2003(3):661-8.

12. Fagan JJ, Jacobs M. Survey of ENT services in Africa: need for a comprehensive intervention. Glob Health Action. 2009; 2: 1-7

13. Kaf WA, Masterson CG, Dion N, Berg SL, Abdelhakiem MK. Optimizing Otoscopy Competency in Audiology Students through Supplementary Otoscopy Training. J Am Acad Audiol. 2013; 859-866

14. Munangatire T, Naidoo N. Exploring of high-fidelity simulation: Nurse educators' perceptions and experiences at a school of nursing in a resource-limited setting. Afr. J. Health Prof. Educ. 2017; 9: 44-47

15. Health Professional Council of south Africa, (2014). Regulations relating to the undergraduate curricula and professional examination in Audiology. http://www.hpcsa.co.za/Uploads/editor/UserFiles/downloads/ legislations/regulations/s/h/regulations/regulations_gnr_106_2014.pdf. Accessed 02 July 2017

16. Biagio L, Swanepoel de W, Laurent C, Lundberg T. Video-otoscopy recordings for diagnosis of childhood ear disease using telehealth at primary health care level. J Telemed Telecare. 2014; 20: 300-6

17. Sullivan RF. Video Otoscopy in Audiological Practice. J Am Acad Audiol. 1997; 8: 447-467

18. Sebothoma B, Khoza-Shangase K. A comparison between video otoscopy and standard tympanometry findings in adults living with human immunodeficiency virus in South Africa. S Afr J Commun Disord. 2018; 65; 1-7

19. Jaisinghani VJ, Hunter LL, Li Y, Margolis RH. Quantitative Analysis of Tympanic Membrane Disease Using Video-Otoscopy. Laryngoscope. 2000; 110: $1726-1730$

20. Myburgh HC, Van Zijl WH, Swanepoel DW, Hellström, Laurentm C. Otitis Media Diagnosis for Developing Countries Using Tympanic Membrane Image-Analysis. EBiomedicine. 2016; 5: 156-160

21. Leedy PD, Ormrod JE. Practical research: Planning and Design. 10th Ed. Boston, MA: Pearson Education. 2013

22. Satake EB. Statistical Methods and Reasoning for the Clinical Science: Evidence based Practice. San Diego, CA: Plural Publishing, Inc. 2015

23. You P, Chahine S, Husein M. Improving learning and confidence through small group, structured otoscopy teaching: a prospective interventional study. J Otolaryngol Head Neck Surg. 2017; 46:1-6

24. Hughes J, Wilson WJ, MacBean N, Hill AE. Simulated patients versus seminars to train case history and feedback skills in audiology students. A randomized controlled trial. Int J Audiol. 2016; 55: 758-764

25. Wilson WJ, Schmulian D, Sher A, Morris S, Hill AE. Student perceptions of two simulated learning environments in paediatric audiology. Int J Audiol. 2020; 59:16-23

26. Von Buchwald JH, Frendø M, Guldager MJ, Melchiors J, Andersen SA. Content validity evidence for a simulation-based test of handheld otoscopy skills. Eur Arch Otorhinolaryngol. 2021; 278:2313-2320

27. Trecca EMC, Lonigro A, Gelardi M, Kim B, Cassano M. Mobile Applications in Otolaryngology: A Systematic Review of the Literature, Apple App Store and 
the Google Play Store. Ann Otol Rhinol Laryngol. 2021;130(1):78-91. doi: https://doi.org/10.1177/0003489420940350. Epub 2020 Jul 13. PMID: 32659107

28. Bhavana K, Ahmad M, Sharma P. Smartphone Otoscopy Sans Attachment: A Paradigm Shift in Diagnosing Ear Pathologies. Otolaryngol Head Neck Surg. 2018; DOl:https://doi.org/10.1177/2473973974X18786496

29. Myburgh HC, Jose S, Swanepoel DW, Laurent C. Otitis Media Diagnosis for Developing Countries Using Tympanic Membrane Image-Analysis. Biomed Signal Process Control. 2018; 39:34-52

30. Parma B, Beukes E, Rajasingam S. The impact of COVID-19 on provision of UK audiology services \& on attitudes towards delivery of telehealth services. Int J Audiol. 2021; DOl: https://doi.org/10.1080/14992027.2021.1921292

31. Khoza-Shangase K, Moroe N, Neille J. Speech-language pathology and audiology in South Africa: clinical training and service in the era of COVID19. Int J Telerehabil. 2021; 13: (10.5195/ijt.2021.6376)

32. Sebothoma B, Khoza-Shangase K. A comparison between video otoscopy and standard tympanometry findings in adults living with human immunodeficiency virus (HIV) in South Africa. S Afr J Commun Disord. 2018; 65: a591. https://doi.org/10.4102/sajcd.v65i1.591

33. Binol H, Niazi MKK, Essig G, Shah J, Mattingly JK, Harris MS, Elmaraghy C, Teknos T, Taj-Schaal N, Yu L, Gurcan MN, Moberly AC. Digital Otoscopy Videos Versus Composite Images: A Reader Study to Compare the Accuracy of ENT Physicians. Laryngoscope. 2020 Nov 10. doi: https://doi.org/10.1002/ lary.29253. Epub ahead of print. PMID: 33170529

34. Pillay M, Tiwari R, Kathard H, Chikte U. (2020). Sustainable workforce: South African Audiologists and Speech Therapists. Hum Resour Health. 2020; 18:113

\section{Publisher's Note}

Springer Nature remains neutral with regard to jurisdictional claims in published maps and institutional affiliations.

Ready to submit your research? Choose BMC and benefit from:

- fast, convenient online submission

- thorough peer review by experienced researchers in your field

- rapid publication on acceptance

- support for research data, including large and complex data types

- gold Open Access which fosters wider collaboration and increased citations

- maximum visibility for your research: over $100 \mathrm{M}$ website views per year

At $\mathrm{BMC}$, research is always in progress.

Learn more biomedcentral.com/submissions 